\title{
Nakajima Varieties and Repetitive Algebras
}

\author{
To the memory of Dieter Happel
}

by

\author{
Bernard LECLERC and Pierre-Guy Plamondon
}

\begin{abstract}
We realize certain graded Nakajima varieties of finite Dynkin type as orbit closures of repetitive algebras of Dynkin quivers. As an application, we show that the perverse sheaves introduced by Nakajima to describe irreducible characters of quantum loop algebras are isomorphic to the intersection cohomology sheaves of these orbit closures.
\end{abstract}

2010 Mathematics Subject Classification: 14L24, 16G20, $17 \mathrm{~B} 37$.

Keywords: Nakajima variety, repetitive algebra, quantum loop algebra, quiver, orbit closure, intersection cohomology.

\section{Contents}

1 Introduction 532

2 Nakajima varieties and representations of algebras 533

2.1 Varieties of representations 533

2.2 Nakajima's varieties 534

2.3 Projectivization 537

2.4 An isomorphism of varieties 538

3 Orbits and strata 541

3.1 Repetitive algebras 541

3.2 The Grothendieck group for repetitive algebras 547

3.3 A bijection between strata and orbits 550

4 Composition multiplicities for quantum loop algebras 556

4.1 Quantum loop algebras and $q$-characters 556

4.2 Nakajima varieties and composition multiplicities 557

4.3 Parametrization of irreducible modules 558

4.4 Composition multiplicities 559

References 560

Communicated by H. Nakajima. Received September 22, 2012. Revised January 18, 2013.

B. Leclerc: Normandie Univ. France, UNICAEN, LMNO, F-14032 Caen, France; CNRS UMR 6139, F-14032 Caen, France; and Institut Universitaire de France; e-mail: bernard.leclerc@unicaen.fr

P.-G. Plamondon: Université de Paris Sud XI, UMR 8628 du CNRS, Laboratoire de Mathématique - Bât. 425, 91405 Orsay Cedex, France;

e-mail: pierre-guy.plamondon@math.u-psud.fr

(C) 2013 Research Institute for Mathematical Sciences, Kyoto University. All rights reserved. 


\section{$\S 1$. Introduction}

Let $U_{q}(L \mathfrak{g})$ be the quantum loop algebra of a simple Lie algebra $\mathfrak{g}$ of Dynkin type $\mathbb{A}, \mathbb{D}$ or $\mathbb{E}$, introduced by Drinfeld and Jimbo. This is a $\mathbb{C}$-algebra, and we assume that the quantum parameter $q \in \mathbb{C}^{*}$ is not a root of unity. The category of finite-dimensional representations of $U_{q}(L \mathfrak{g})$ has been studied by many authors $[1,5,6,7,9,17]$.

In type $\mathbb{A}$, a geometric construction of standard and irreducible $U_{q}(L \mathfrak{g})$ modules was given by Ginzburg and Vasserot [7, 22]. In particular, they showed that the composition multiplicities of standard modules can be expressed in terms of the local intersection cohomology of graded nilpotent orbit closures. This theory was extended by Nakajima to all simply-laced types. To do so, he replaced the graded nilpotent orbit closures by certain graded versions $\mathfrak{M}_{0}^{\bullet}(V, W)$ of the quiver varieties $\mathfrak{M}_{0}(V, W)$ previously introduced in his geometric construction of irreducible finite-dimensional $\mathfrak{g}$-modules. Nakajima varieties are defined as quotients in geometric invariant theory, and in general they do not have any other more explicit description.

Let $Q$ be a Dynkin quiver of the same type as $\mathfrak{g}$. It was recently observed [11] that the orbit closures of $Q$ are isomorphic to some particular Nakajima graded quiver varieties $\mathfrak{M}_{0}^{\bullet}(V, W)$. In this paper we generalize this observation and show that all the orbit closures of the repetitive algebra [12] of the path algebra $\mathbb{C} Q$ are isomorphic to certain varieties $\mathfrak{M}_{0}^{\bullet}(V, W)$. The repetitive algebra $\widehat{A}$ of an algebra $A$, first introduced by Hughes and Waschbüsch, is a selfinjective infinite-dimensional algebra, defined as a kind of infinite matrix algebra (its precise definition is recalled in §3.1). It was shown by Happel that, if $A$ has finite global dimension, the stable module category of $\widehat{A}$ is equivalent to the derived category of $A$. When $Q$ is a linearly oriented Dynkin quiver of type $\mathbb{A}$, the orbit closures of the repetitive algebra $\widehat{\mathbb{C Q}}$ coincide with the graded nilpotent orbit closures of Ginzburg and Vasserot. Our result may thus be regarded as an explicit description, similar to that of Ginzburg and Vasserot, of a large collection of graded Nakajima varieties $\mathfrak{M}_{0}^{\bullet}(V, W)$.

Although we only realize in this way varieties corresponding to some particular $W$ 's, we get enough of them to express all the irreducible characters of $U_{q}(L \mathfrak{g})$ in terms of the local intersection cohomology of the orbit closures of $\widehat{\mathbb{C Q}}$. Recall that the perverse sheaves used by Lusztig [15] in his geometric construction of the canonical basis of $U_{q}^{+}(\mathfrak{g})$ are precisely the intersection cohomology sheaves of the orbit closures of $Q$. This description of the canonical basis was inspired by Ringel's theorem [20] stating that $U_{q}^{+}(\mathfrak{g})$ is isomorphic to the Hall algebra of $Q$. Similarly our result shows that the perverse sheaves used by Nakajima in his geometric construction of the canonical basis of the Grothendieck ring of $U_{q}(L \mathfrak{g})$ 
are essentially the intersection cohomology sheaves of the orbit closures of $\widehat{\mathbb{C Q}}$. This was inspired by the recent result of [11], stating that the $t$-deformed Grothendieck ring is isomorphic to Töen's Hall algebra of the derived category of $Q$, which, by Happel's theorem, is isomorphic to Töen's algebra of the stable category of $\widehat{\mathbb{C Q}}$.

Another interesting application of our results is that we can apply Nakajima's well-developed theory to get new information on the orbit closures of repetitive algebras of Dynkin type. For instance we obtain desingularizations of these orbit closures with favorable properties, like the vanishing of odd cohomology groups of fibers. Also, using Nakajima's algorithm to compute the irreducible characters of $U_{q}(L \mathfrak{g})$ [18], we can in principle calculate the local intersection cohomology of these orbit closures.

Our paper is structured as follows. In $\S 2$, we recall the definition of the graded Nakajima varieties $\mathfrak{M}_{0}^{\bullet}(V, W)$ and $\mathfrak{M}_{0}^{\bullet}(W)$, as well as the stratification of $\mathfrak{M}_{0}^{\bullet}(W)$ by the strata $\mathfrak{M}_{0}^{\bullet r e g}(V, W)$. We then show that the variety $\mathfrak{M}_{0}^{\bullet}(W)$ is isomorphic to the variety of representations with dimension vector $\mathbf{d}$ of a certain finitedimensional algebra $e_{\Sigma} \Lambda e_{\Sigma}$ obtained by projectivization (Theorem 2.4). Here $\mathbf{d}$ is the graded dimension of $W$. If $W$ is arbitrary, $e_{\Sigma} \Lambda e_{\Sigma}$ looks rather complicated. But we show in $\S 3$ that for certain special choices of $W$, the algebra $e_{\Sigma} \Lambda e_{\Sigma}$ is isomorphic to the repetitive algebra $\widehat{A}$ of $A=\mathbb{C} Q$ (Lemma 3.6). Moreover, in this case, we show (Theorem 3.14) that Nakajima's stratification of $\mathfrak{M}_{0}^{\bullet}(W)$ is identical to the usual orbit stratification of $\operatorname{rep}_{\mathbf{d}}(\widehat{A})$ (recall that since $Q$ is of Dynkin type, $\widehat{A}$ is locally of finite representation type). The precise correspondence between isoclasses $N$ of $\widehat{A}$-modules of dimension $\mathbf{d}$ and strata $\mathfrak{M}_{0}^{\bullet r e g}(V, W)$ is described in Corollary 3.15. Finally in $\S 4$, we apply our results to the representation theory of quantum loop algebras $U_{q}(L \mathfrak{g})$. We start by recalling the definition of the tensor category $\mathcal{C}_{\mathbb{Z}}$ of finite-dimensional $U_{q}(L \mathfrak{g})$-modules, and we state Nakajima's theorem giving a geometric description of the composition multiplicities of standard modules of $\mathcal{C}_{\mathbb{Z}}$. Then we deduce from Theorem 3.14 that there is a natural one-to-one correspondence between the isomorphism classes of objects in the stable category $\underline{\bmod } \widehat{A}$ and the isomorphism classes of simple objects in $\mathcal{C}_{\mathbb{Z}}$ (Proposition 4.2 ). We conclude with a description of the composition multiplicities of standard modules in terms of the intersection cohomology of the $\widehat{A}$-orbit closures.

\section{§2. Nakajima varieties and representations of algebras}

\section{$\S 2.1$. Varieties of representations}

We will work over the field $\mathbb{C}$ of complex numbers. Let $Q=\left(Q_{0}, Q_{1}\right)$ be a quiver with vertex set $Q_{0}$ and arrow set $Q_{1}$. Let $s, t: Q_{1} \rightarrow Q_{0}$ be maps taking each arrow $a$ to its source $s(a)$ or its target $t(a)$. The number of vertices may be infinite, but 
we will assume that the number of arrows incident with any given vertex is finite. Let $A:=\mathbb{C} Q / R$ be the quotient of the path algebra of $Q$ by an ideal $R$. We will always assume that $R$ is contained in the two-sided ideal generated by the arrows of $Q$. Then $A$ is an associative algebra which has no unit if the number of vertices of $Q$ is infinite. For any vertex $i$ of $Q$, let $e_{i}$ be the trivial path at $i$.

A representation of $(Q, R)$ is a pair $\left(\left(V_{i}\right)_{i \in Q_{0}},\left(\varphi_{a}\right)_{a \in Q_{1}}\right)$, where each $V_{i}$ is a vector space over $\mathbb{C}$ and each $\varphi_{a}$ is a linear map from $V_{s(a)}$ to $V_{t(a)}$ such that all polynomials in the $\varphi_{a}$ corresponding to elements in $R$ vanish. The representations of $(Q, R)$ form a category. This category is equivalent to the category of left modules $M$ over $\mathbb{C} Q$ which have the property that $M=\bigoplus_{i \in Q_{0}} e_{i} M$.

Let $\mathbf{d} \in \mathbb{N}^{Q_{0}}$ be a finite dimension vector, that is, such that the sum of its entries $d_{i}$ for $i \in Q_{0}$ is finite. The variety of representations of $A$ of dimension vector $\mathbf{d}$ is the closed subset $\operatorname{rep}_{\mathbf{d}}(A)$ of

$$
\bigoplus_{a \in Q_{1}} \operatorname{Hom}_{\mathbb{C}}\left(\mathbb{C}^{d_{s(a)}}, \mathbb{C}^{d_{t(a)}}\right)
$$

which vanishes on all polynomials defined by the relations $R$. Its points are in bijection with the representations of $A$ with dimension vector $\mathbf{d}$ and underlying vector space $\prod_{i \in Q_{0}} \mathbb{C}^{d_{i}}$. It is a finite-dimensional affine algebraic variety.

The affine algebraic group

$$
G_{\mathbf{d}}:=\prod_{i \in Q_{0}} \operatorname{GL}\left(\mathbb{C}^{d_{i}}\right)
$$

acts on $\operatorname{rep}_{\mathbf{d}}(A)$ by base change at every vertex. The $G_{\mathbf{d}^{-}}$-orbits are in bijection with the isomorphism classes of $A$-modules with dimension vector $\mathbf{d}$.

\section{§2.2. Nakajima's varieties}

Let $Q$ be a quiver of Dynkin type $\mathbb{A}, \mathbb{D}$ or $\mathbb{E}$. For $i, j$ in $Q_{0}$, we write $i \sim j$ if there is in $Q_{1}$ an arrow $i \rightarrow j$ or an arrow $j \rightarrow i$. A height function on $Q$ is a map

$$
\xi: Q_{0} \rightarrow \mathbb{Z}
$$

such that for any arrow $i \rightarrow j$ in $Q$, we have $\xi_{j}=\xi_{i}-1$. Since $Q$ is a tree, there exists a height function on it.

Define the repetition quiver $\widehat{Q}$ thus:

- the set of vertices of $\widehat{Q}$ is $\widehat{Q}_{0}=\left\{(i, p) \in Q_{0} \times \mathbb{Z} \mid p-\xi_{i} \in 2 \mathbb{Z}\right\}$;

- for any arrow $c: i \rightarrow j$ in $Q$ and any $(i, n) \in \widehat{Q}_{0}$, there is an arrow $(c, n)$ : $(i, n) \rightarrow(j, n-1)$ and an arrow $(\bar{c}, n-1):(j, n-1) \rightarrow(i, n-2)$ in $\widehat{Q}$. 
Now, let $\widehat{\Gamma}$ be the quiver which contains $\widehat{Q}$ as a full subquiver, and which has additional vertices and arrows as follows:

- one additional vertex for each element of $\left\{(i, p) \in Q_{0} \times \mathbb{Z} \mid p-\xi_{i} \in 1+2 \mathbb{Z}\right\}$. In particular, the set of vertices of $\widehat{\Gamma}$ is $Q_{0} \times \mathbb{Z}$;

- additional arrows $a_{(i, n+1)}:(i, n+1) \rightarrow(i, n)$ and $b_{(i, n)}:(i, n) \rightarrow(i, n-1)$ for every $(i, n) \in \widehat{Q}_{0}$.

We will study representations of $\widehat{\Gamma}$ satisfying the following set of relations:

$$
a_{(i, n-1)} b_{(i, n)}+\sum_{c: i \rightarrow j}(\bar{c}, n-1)(c, n)-\sum_{c: j \rightarrow i}(c, n-1)(\bar{c}, n)=0, \quad(i, n) \in \widehat{Q}_{0} .
$$

Let $R$ be the ideal generated by these relations. Let $\Lambda$ be the quotient of $\mathbb{C} \widehat{\Gamma}$ by $R$.

Let $\mathbf{d}$ be a finite dimension vector on $\widehat{\Gamma}$. We will use the following notation:

$$
V=\bigoplus_{(i, n) \in \widehat{Q}_{0}} \mathbb{C}^{d_{(i, n)}}, \quad W=\bigoplus_{(i, n) \in \widehat{\Gamma}_{0} \backslash \widehat{Q}_{0}} \mathbb{C}^{d_{(i, n)}}
$$

where $V$ is a $\widehat{Q}_{0}$-graded vector space, and $W$ is a $\left(\widehat{\Gamma}_{0} \backslash \widehat{Q}_{0}\right)$-graded vector space. Then the variety of representations $\operatorname{rep}_{\mathbf{d}}(\Lambda)$ is a closed subset of the direct sum of these three vector spaces:

$$
\begin{gathered}
L^{\bullet}(V, W)=\bigoplus_{(i, n) \in \widehat{Q}_{0}} \operatorname{Hom}_{\mathbb{C}}\left(V_{(i, n)}, W_{(i, n-1)}\right), \\
L^{\bullet}(W, V)=\bigoplus_{(i, n) \in \widehat{Q}_{0}} \operatorname{Hom}_{\mathbb{C}}\left(W_{(i, n+1)}, V_{(i, n)}\right), \\
E^{\bullet}(V)=\bigoplus_{i \rightarrow j,(i, n) \in \widehat{Q}_{0}} \operatorname{Hom}_{\mathbb{C}}\left(V_{(i, n)}, V_{(j, n-1)}\right) \oplus \bigoplus_{i \rightarrow j,(j, m) \in \widehat{Q}_{0}} \operatorname{Hom}_{\mathbb{C}}\left(V_{(j, m)}, V_{(i, m-1)}\right) .
\end{gathered}
$$

The variety $\operatorname{rep}_{\mathbf{d}}(\Lambda)$ will also be denoted by $\Lambda^{\bullet}(V, W)$ when we want to emphasize this decomposition.

The affine algebraic group

$$
G_{V}:=\prod_{(i, n) \in \widehat{Q}_{0}} \operatorname{GL}\left(V_{(i, n)}\right)
$$

acts naturally on $\Lambda^{\bullet}(V, W)$ by base change at vertices $(i, n) \in \widehat{Q}_{0}$. Define the affine quotient

$$
\mathfrak{M}_{0}^{\bullet}(V, W):=\Lambda^{\bullet}(V, W) / / G_{V}:=\operatorname{Spec} \mathbb{C}\left[\Lambda^{\bullet}(V, W)\right]^{G_{V}}
$$


If $V \subset V^{\prime}$ as $\widehat{Q}_{0}$-graded vector spaces, then there is a natural closed embedding $\mathfrak{M}_{0}^{\bullet}(V, W) \subset \mathfrak{M}_{0}^{\bullet}\left(V^{\prime}, W\right)$. Define

$$
\mathfrak{M}_{0}^{\bullet}(W)=\bigcup_{V} \mathfrak{M}_{0}^{\bullet}(V, W)
$$

It is an affine variety on which the algebraic group

$$
G_{W}:=\prod_{(i, n) \in \widehat{\Gamma}_{0} \backslash \widehat{Q}_{0}} \operatorname{GL}\left(W_{(i, n)}\right)
$$

acts by base change at vertices $(i, n) \in \widehat{\Gamma}_{0} \backslash \widehat{Q}_{0}$. Nakajima proves (see for instance $[19$, Section 3]) that there is a stratification

$$
\mathfrak{M}_{0}^{\bullet}(W)=\bigsqcup_{V} \mathfrak{M}_{0}^{\bullet \text { reg }}(V, W)
$$

where $\mathfrak{M}_{0}^{\bullet r e g}(V, W)$ is the open subset of $\mathfrak{M}_{0}^{\bullet}(V, W)$ parametrizing the closed free $G_{V}$-orbits. He also proves that a necessary condition for $\mathfrak{M}_{0}^{\bullet r e g}(V, W)$ to be nonempty is that

$$
\operatorname{dim} W_{(i, n)}-\operatorname{dim} V_{(i, n+1)}-\operatorname{dim} V_{(i, n-1)}+\sum_{j \sim i} \operatorname{dim} V_{(j, n)} \geq 0
$$

for all $(i, n) \in \Gamma_{0} \backslash Q_{0}$. In that case, the pair $(V, W)$ is called dominant.

Similarly, we will say that a representation $N$ of $\Lambda$ is dominant if the associated pair $(V, W)$ is dominant. The representation $N$ is stable if any graded subspace of $V$ stable under the action of all $(c, n)$ and $(\bar{c}, n+1)$ and vanishing under the action of all $b_{(i, n)}$ is the zero subspace. In other words, $N$ is stable if it has no non-zero subrepresentations supported on $V$.

The stratum $\mathfrak{M}_{0}^{\text {oreg }}(V, W)$ is non-empty if, and only if, there exists a stable representation $N$ whose underlying graded vector space is $(V, W)$, and $(V, W)$ is dominant.

Example 2.1. Consider the quiver $Q$ of type $\mathbb{A}_{4}$ given by

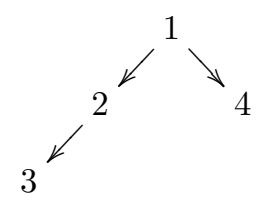

with height function defined by $\xi_{1}=2, \xi_{2}=\xi_{4}=1$ and $\xi_{3}=0$. Then the points of $\Lambda^{\bullet}(V, W)$ are given by collections of morphisms which can be organized as in the following picture: 


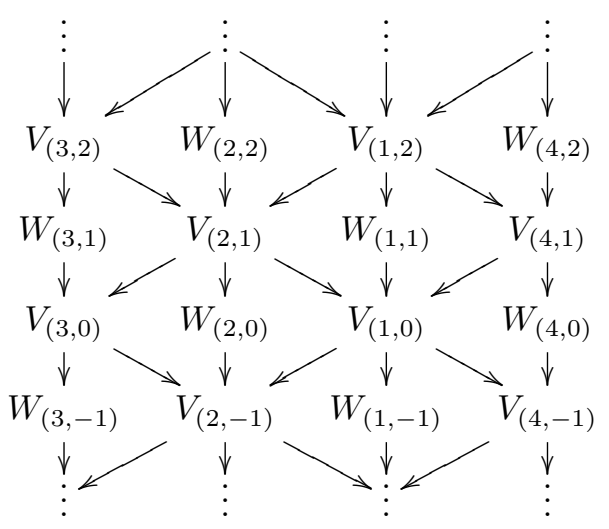

The quiver $Q$ is found in this picture by taking the full subquiver whose vertices are $V_{(1,2)}, V_{(2,1)}, V_{(3,0)}$ and $V_{(4,1)}$. The quiver whose arrows and vertices are all the $V$ 's and $W$ 's is $\widehat{\Gamma}$; the full subquiver whose vertices are the $V$ 's is $\widehat{Q}$.

\section{§2.3. Projectivization}

Let $\widehat{\Gamma}, R$ and $\Lambda$ be as above. Let $\Sigma$ be a subset of $\widehat{\Gamma}_{0}$. Define $\widehat{\Gamma}_{\Sigma}$ as the full subquiver of $\widehat{\Gamma}$ whose vertices are those $x$ in $\widehat{\Gamma}_{0}$ such that there exist two vertices $w$ and $y$ in $\Sigma$ and a path from $w$ to $y$ passing through $x$. (One may think of $\widehat{\Gamma}_{\Sigma}$ as a kind of convex hull of $\Sigma$.)

Let $R_{\Sigma}:=R \cap \mathbb{C} \widehat{\Gamma}_{\Sigma}$, and define $\Lambda_{\Sigma}:=\mathbb{C} \widehat{\Gamma}_{\Sigma} / R_{\Sigma}$. If $\mathbf{d}$ is a dimension vector supported on $\widehat{\Gamma}_{\Sigma}$, then $\operatorname{rep}_{\mathbf{d}}(\Lambda) \cong \operatorname{rep}_{\mathbf{d}}\left(\Lambda_{\Sigma}\right)$. Note that if $\Sigma$ is finite, then $\widehat{\Gamma}_{\Sigma}$ only has a finite number of vertices, and $\Lambda_{\Sigma}$ is a finite-dimensional algebra with unit.

For the rest of the section, we will assume that $\Sigma$ is finite, and that

$$
\Sigma \subset \widehat{\Gamma}_{0} \backslash \widehat{Q}_{0} .
$$

Define the idempotent

$$
e_{\Sigma}:=\sum_{x \in \Sigma} e_{x}
$$

and consider the algebra $e_{\Sigma} \Lambda e_{\Sigma}=e_{\Sigma} \Lambda_{\Sigma} e_{\Sigma}$. We will list here some facts concerning this algebra, taken mainly from Chapter II.2 of [4].

The algebra $e_{\Sigma} \Lambda_{\Sigma} e_{\Sigma}$ is a finite-dimensional algebra with unit $e_{\Sigma}$, and the $e_{x}$, for $x$ in $\Sigma$, form a complete set of pairwise orthogonal primitive idempotents. It is isomorphic as an algebra to $\operatorname{End}_{\Lambda_{\Sigma}}\left(\Lambda_{\Sigma} e_{\Sigma}\right)^{\mathrm{op}}$. From this isomorphism, we see that we have a functor

$$
\operatorname{Hom}_{\Lambda_{\Sigma}}\left(\Lambda_{\Sigma} e_{\Sigma}, ?\right): \bmod \Lambda_{\Sigma} \rightarrow \bmod e_{\Sigma} \Lambda_{\Sigma} e_{\Sigma}
$$


which is exact since $\Lambda_{\Sigma} e_{\Sigma}$ is a projective $\Lambda_{\Sigma}$-module. The process of applying this functor is called projectivization in [4].

Let $\operatorname{pres}\left(\Lambda_{\Sigma} e_{\Sigma}\right)$ be the full subcategory of $\bmod \Lambda_{\Sigma}$ consisting of modules $M$ which admit a projective presentation

$$
P_{1} \rightarrow P_{0} \rightarrow M \rightarrow 0,
$$

with $P_{1}$ and $P_{0}$ in add $\left(\Lambda_{\Sigma} e_{\Sigma}\right)$.

Proposition 2.2 (Proposition II.2.5 of [4]). The functor $\operatorname{Hom}_{\Lambda_{\Sigma}}\left(\Lambda_{\Sigma} e_{\Sigma}, ?\right)$ restricts to an equivalence of categories

$$
\operatorname{pres}\left(\Lambda_{\Sigma} e_{\Sigma}\right) \rightarrow \bmod e_{\Sigma} \Lambda_{\Sigma} e_{\Sigma} .
$$

Let $M$ be a $\Lambda_{\Sigma}$-module. The dimension vector of its image in $\bmod e_{\Sigma} \Lambda_{\Sigma} e_{\Sigma}$ is obtained from $\operatorname{dim} M$ by forgetting the components which are not in $\Sigma$. This is seen from the isomorphisms

$$
e_{x} \operatorname{Hom}_{\Lambda_{\Sigma}}\left(\Lambda_{\Sigma} e_{\Sigma}, M\right) \cong e_{x} \operatorname{Hom}_{\Lambda_{\Sigma}}\left(\Lambda_{\Sigma}, M\right) \cong e_{x} M .
$$

For any dimension vector $\mathbf{d}$ on $\widehat{\Gamma}$, we write $\mathbf{d}=\mathbf{d}_{V}+\mathbf{d}_{W}$, where $\mathbf{d}_{V}$ is supported on $\widehat{Q}_{0}$ and $\mathbf{d}_{W}$ is supported on $\widehat{\Gamma}_{0} \backslash \widehat{Q}_{0}$. The above discussion gives us the following, which we will use later.

Corollary 2.3. Let $M$ be a module over $e_{\Sigma} \Lambda_{\Sigma} e_{\Sigma}$. Then there exists a $\Lambda_{\Sigma}$-module $L$ such that $\operatorname{Hom}_{\Lambda_{\Sigma}}\left(\Lambda_{\Sigma} e_{\Sigma}, L\right)$ is isomorphic to $M$. Its dimension vector $\mathbf{d}=$ $\mathbf{d}_{V}+\mathbf{d}_{W}$ is such that $\mathbf{d}_{W}$ is the dimension vector of $M$.

\section{$\S 2.4$. An isomorphism of varieties}

In this section, we prove the following result, which is motivated by Theorem 9.11 of [11]. Let $\Sigma$ be a finite subset of $\widehat{\Gamma}_{0}$ satisfying the assumption (2), and let $\mathbf{d}$ be a dimension vector supported on $\Sigma$. Define

$$
W^{\mathbf{d}}:=\bigoplus_{(i, n) \in \Sigma} \mathbb{C}^{d_{(i, n)}}
$$

Theorem 2.4. There is a $G_{W \mathrm{~d}}$-equivariant isomorphism of varieties

$$
\Psi: \mathfrak{M}_{0}^{\bullet}\left(W^{\mathbf{d}}\right) \rightarrow \operatorname{rep}_{\mathbf{d}}\left(e_{\Sigma} \Lambda e_{\Sigma}\right) .
$$

Proof. We shall write for short $e$ instead of $e_{\Sigma}$, and $W$ instead of $W^{\mathbf{d}}$. We proceed in two steps. We will first show that there is a closed immersion $\Psi$, in much the same way as in Proposition 9.4 of [11]. Then we will prove that $\Psi$ is surjective.

Notice first that $e \Lambda e=e \Lambda_{\Sigma} e$ is a finite-dimensional algebra with unit. Let $\left\{\alpha_{k}\right\}_{k=1}^{r}$ be a finite set of generators of $e \Lambda e$. We assume that each $\alpha_{k}$ lies in some 
$e_{x} \Lambda e_{y}$ for vertices $x$ and $y$ of $\widehat{\Gamma} \backslash \widehat{Q}$. Then $\operatorname{rep}_{\mathbf{d}}(e \Lambda e)$ is a closed subset of the affine space

$$
\bigoplus_{\alpha_{k} \in e_{x} \Lambda e_{y}} \operatorname{Hom}_{\mathbb{C}}\left(W_{x}, W_{y}\right)
$$

Its coordinate ring can therefore be expressed as a quotient of a polynomial ring $\mathbb{C}\left[\theta_{i, j}^{k}\right]_{i, j, k}$, where $\theta_{i, j}^{k}$ should be seen as the function sending a linear map in $\operatorname{Hom}_{\mathbb{C}}\left(W_{x}, W_{y}\right)$ to the $(i, j)$ th coordinate of this map written in matrix form for some fixed bases of $W_{x}$ and $W_{y}$.

Now fix some $\widehat{Q}_{0}$-graded vector space $V$. Each $\alpha_{k}$ is an element of some $e_{x} \Lambda e_{y}$; it is thus a linear combination of paths in $\widehat{\Gamma}$ from $x$ to $y$. For a representation in $\Lambda \bullet(V, W)$, this linear combination of paths corresponds to a linear combination of compositions of linear maps, each composition going from $W_{x}$ to $W_{y}$. Let $\psi_{i, j}^{k}$ be the function in $\mathbb{C}\left[\Lambda^{\bullet}(V, W)\right]^{G_{V}}$ corresponding to the $(i, j)$ th coordinate of this map from $W_{x}$ to $W_{y}$ written in matrix form. Define an algebra morphism by

$$
\mathbb{C}\left[\operatorname{rep}_{\mathbf{d}} e \Lambda e\right] \rightarrow \mathbb{C}\left[\Lambda^{\bullet}(V, W)\right]^{G_{V}}, \quad \theta_{i, j}^{k} \mapsto \psi_{i, j}^{k} .
$$

According to [19] (see also [14], [16]), the functions $\psi_{i, j}^{k}$ generate $\mathbb{C}\left[\Lambda^{\bullet}(V, W)\right]^{G_{V}}$. We have thus defined a surjective morphism between coordinate rings, which yields a closed immersion $\Psi_{V}: \mathfrak{M}_{0}^{\bullet}(V, W) \rightarrow \operatorname{rep}_{\mathbf{d}} e \Lambda e$. Since $\mathfrak{M}_{0}^{\bullet}(W)$ is the union of all $\mathfrak{M}_{0}^{\bullet}(V, W)$, we get a closed immersion $\Psi: \mathfrak{M}_{0}^{\bullet}(W) \rightarrow \operatorname{rep}_{\mathbf{d}} e \Lambda e$.

We now prove that $\Psi$ is surjective. The action of $\Psi$ can be understood using the following diagram:

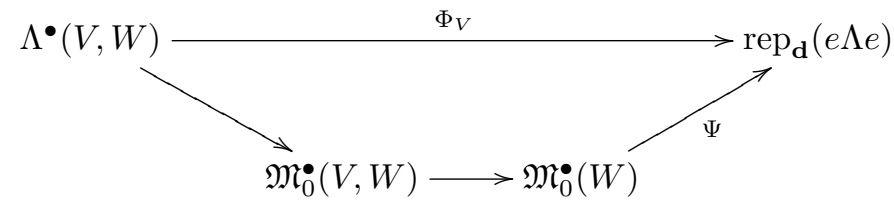

Here, $\Phi_{V}$ sends a representation $M$ of $\Lambda$ in $\Lambda^{\bullet}(V, W)$ to a representation of $e \Lambda e$ isomorphic to $\operatorname{Hom}_{\Lambda_{\Sigma}}\left(\Lambda_{\Sigma} e, M\right)$. To prove that $\Psi$ is surjective, it is thus sufficient to prove that for any representation $N$ in $\operatorname{rep}_{\mathbf{d}}(e \Lambda e)$, there exists a $V$ and a representation $M$ in $\Lambda^{\bullet}(V, W)$ such that $N \cong \Phi_{V}(M) \cong \operatorname{Hom}_{\Lambda_{\Sigma}}\left(\Lambda_{\Sigma} e, M\right)$. But such $V$ and $M$ always exist thanks to Corollary 2.3. Thus $\Psi$ is surjective. Finally, the definition of $\Psi$ makes it $G_{W}$-equivariant. The theorem is proved.

Remark 2.5. The same result was proved in [11, Proposition 9.8] for a restricted choice of $\Sigma$ determined by the representation theory of the quiver $Q$.

Example 2.6. Consider the quiver $Q=1 \rightarrow 2$ of type $\mathbb{A}_{2}$, with height function given by $\xi_{1}=1$ and $\xi_{2}=0$. Put $W_{(1,2)}=W_{(2,-1)}=\mathbb{C}$, and set all other $W$ 's to 
zero. We get the picture

$$
\begin{aligned}
& W_{(1,2)}=\mathbb{C} \\
& \downarrow \\
& V_{(1,1)} \\
& \longrightarrow V_{(2,0)} \\
& W_{(2,-1)}=\mathbb{C}
\end{aligned}
$$

In that case, the theorem implies that $\mathfrak{M}_{0}^{\bullet}(W)$ is isomorphic to the variety of representations of $Q$ of dimension vector $(1,1)$.

Example 2.7. Let $Q$ be as in Example 2.6. This time, put

$$
W_{(1,4)}=W_{(1,2)}=W_{(1,0)}=W_{(2,3)}=W_{(2,1)}=W_{(2,-1)}=\mathbb{C} .
$$

We get the picture

$$
\begin{aligned}
& W_{(1,4)}=\mathbb{C}
\end{aligned}
$$

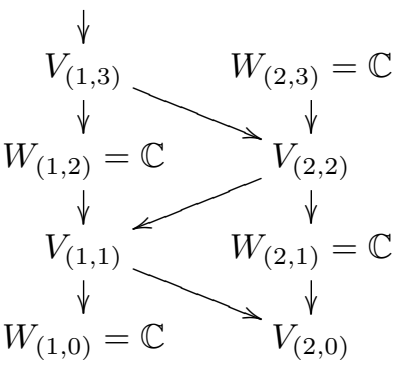

$$
\begin{aligned}
& W_{(2,-1)}=\mathbb{C}
\end{aligned}
$$

The theorem states that $\mathfrak{M}_{0}^{\bullet}(W)$ is isomorphic to the variety of representations of dimension vector $(1,1,1,1,1,1)$ of the quiver

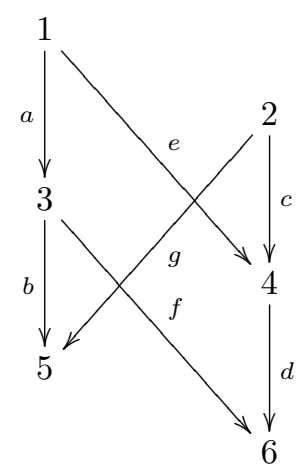

subject to the relation $f a=-d e$. 
These examples illustrate the various forms that the algebra $e_{\Sigma} \Lambda e_{\Sigma}$ can take. In the next section, we will see that, for a suitable choice of $W$, this algebra is the well-studied repetitive algebra.

\section{$\S 3$. Orbits and strata}

\section{$\S 3.1$. Repetitive algebras}

Our main source for this section is Chapter II.2 of [8]. Let $A$ be a finite-dimensional algebra over $\mathbb{C}$. The repetitive algebra $\widehat{A}$ of $A$ is the infinite-dimensional algebra (without unit) defined as a vector space by

$$
\widehat{A}=\bigoplus_{n \in \mathbb{Z}} A \oplus \bigoplus_{n \in \mathbb{Z}} D A
$$

where $D$ is the duality functor $\operatorname{Hom}_{\mathbb{C}}(?, \mathbb{C})$. An element of $\widehat{A}$ will be denoted by $\left(a_{n}, \varphi_{n}\right)_{n}$, with only finitely many of the $a_{n}$ and $\varphi_{n}$ being non-zero. The multiplication rule is given by

$$
\left(a_{n}, \varphi_{n}\right)_{n} \cdot\left(b_{n}, \psi_{n}\right)_{n}=\left(a_{n} b_{n}, a_{n+1} \psi_{n}+\varphi_{n} b_{n}\right)_{n} .
$$

The repetitive algebra $\widehat{A}$ can be interpreted as an "infinite matrix algebra" as follows:

$$
\widehat{A}=\left(\begin{array}{ccccc}
\ddots & & & & \\
\ddots & A & & & \\
& D A & A & & \\
& & D A & A & \\
& & & \ddots & \ddots
\end{array}\right) .
$$

Then the product in $\widehat{A}$ of two infinite matrices of this form is the usual product of matrices, except that the entries of the second subdiagonal in the product are ignored and set to zero.

As shown in [8], $\widehat{A}$ is a selfinjective algebra. Thus its stable module category $\underline{\bmod } \widehat{A}$ is triangulated, and the suspension functor is given by the inverse of the syzygy functor $\Omega$.

Example 3.1. If $A=\mathbb{C} Q$, where $Q$ is the linearly oriented quiver of type $\mathbb{A}_{n}$,

$$
Q=1 \rightarrow 2 \rightarrow \cdots \rightarrow n
$$

then it is easy to see that $\widehat{A}$ is isomorphic to the path algebra of a linearly oriented 
quiver $Q^{\text {repet }}$ with vertex set $\mathbb{Z}$ and the following relations:

- every path of length $>n$ is equal to 0 .

A representation of $\widehat{A}$ of dimension vector $\mathbf{d}=\left(d_{i}\right)$ is the same as a degree 1 endomorphism $x$ of the graded vector space

$$
W^{\mathbf{d}}=\bigoplus_{i \in \mathbb{Z}} \mathbb{C}^{d_{i}}
$$

satisfying $x^{n+1}=0$. The orbits of $\operatorname{rep}_{\mathbf{d}}(\widehat{A})$ are therefore identical to the graded nilpotent orbits of $W$ considered by Ginzburg and Vasserot in [7].

Following J. Schröer [21], we can describe the repetitive algebra $\widehat{\mathbb{C Q}}$ of any quiver $Q$ by a quiver $Q^{\text {repet }}$ with relations as follows:

- the vertices of $Q^{\text {repet }}$ are labelled by $i[n]$, where $i \in Q_{0}$ and $n \in \mathbb{Z}$;

- for any arrow $i \rightarrow j$ of $Q$ and any integer $n$, there is an arrow

$$
i[n] \rightarrow j[n] ;
$$

- for any maximal path $w: i \rightarrow j$ of $Q$ and any integer $n$, there is an arrow

$$
w^{*}[n]: j[n] \rightarrow i[n+1]
$$

(these are called connecting arrows).

Note that in [21], the arrows $w^{*}[n]$ would go from $j[n]$ to $i[n-1]$; this is because our definition for the repetitive algebra, which follows [8], uses different conventions than those in [21]. The relations are obtained in the following way:

- A full path is a path of the form $u[n+1] w^{*}[n] v[n]$, where $w=v u$ is a maximal path in $Q$. Then any path which is not a subpath of a full path is a relation.

- If $w_{1}=x_{1} v u_{1}$ and $w_{2}=x_{2} v u_{2}$ are two maximal paths in $Q$, then the element

$$
u_{1}[n+1] w_{1}^{*}[n] x_{1}[n]-u_{2}[n+1] w_{2}^{*}[n] x_{2}[n]
$$

is a relation.

Example 3.2. Let $Q$ be the quiver of type $\mathbb{A}_{4}$ given by

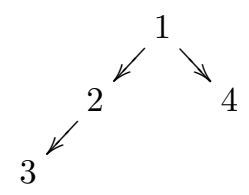

The quiver $Q^{\text {repet }}$ is 


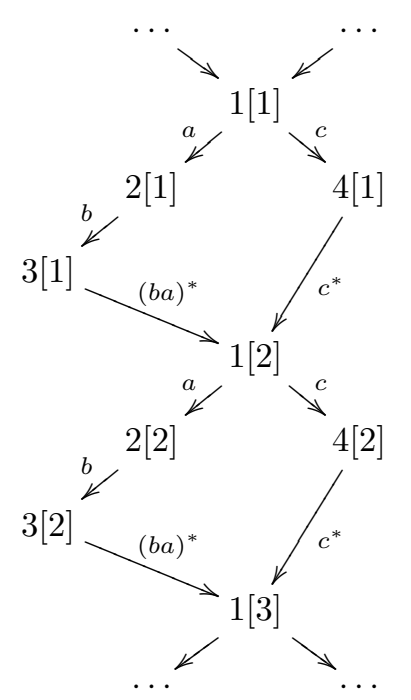

The relations are $c(b a)^{*}=a c^{*}=b a(b a)^{*} b=0,(b a)^{*} b a=c^{*} c$.

Example 3.3. Let $Q$ be the quiver of type $\mathbb{D}_{4}$ given by

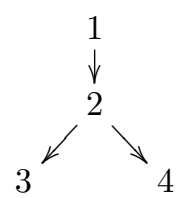

The quiver $Q^{\text {repet }}$ is

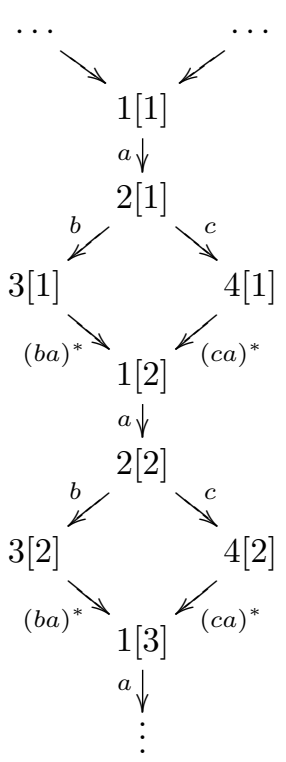


The relations are

$$
c a(b a)^{*}=b a(c a)^{*}=a(c a)^{*} c a=a(b a)^{*} b a=0, \quad(b a)^{*} b=(c a)^{*} c .
$$

We now recall Happel's theorem:

Theorem 3.4 (Theorem II.4.9 of [8]). Assume that A has finite global dimension. Then there is an equivalence of triangulated categories $\mathcal{D}^{b}(\bmod A) \rightarrow \underline{\bmod } \widehat{A}$.

In view of this theorem, the Auslander-Reiten quiver of $\bmod \widehat{A}$ is obtained by adding vertices (corresponding to indecomposable projective-injective $\widehat{A}$-modules) to the Auslander-Reiten quiver of $\mathcal{D}^{b}(\bmod A)$.

Assume now that $A$ is the path algebra of a quiver $Q$ of Dynkin type $\mathbb{A}, \mathbb{D}$ or $\mathbb{E}$. Then the Auslander-Reiten quiver of $\mathcal{D}^{b}(\bmod A)$ is known to be isomorphic to $\widehat{Q}^{\text {op }}$. In order to picture the Auslander-Reiten quiver of $\bmod \widehat{A}$, we must know which irreducible morphisms start or end in each indecomposable projective-injective module.

Let $P$ be an indecomposable projective-injective module. Its socle and top are simple; thus any submodule and any quotient of $P$ are indecomposable. Moreover, using for instance Proposition IV.3.5 of [2], we know that the only irreducible morphisms involving $P$ are the inclusion $\operatorname{rad} P \hookrightarrow P$ and the surjection $P \rightarrow$ $P / \operatorname{soc} P$. Therefore $P$ appears in exactly one mesh of the Auslander-Reiten quiver of $\bmod \widehat{A}$, and this mesh has the form

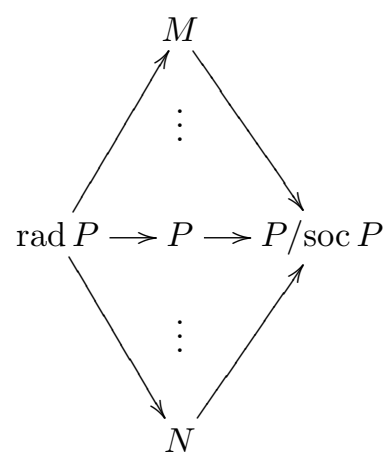

where all modules other than $P$ are non-projective. Thus the Auslander-Reiten quiver of $(\bmod \widehat{A})^{\mathrm{op}}$ is a full subquiver of $\widehat{\Gamma}$.

Example 3.5. We continue Example 3.2. The Auslander-Reiten quiver of $\widehat{\mathbb{C Q}}$ (with arrows going from bottom to top, a convention which will be useful later) is 


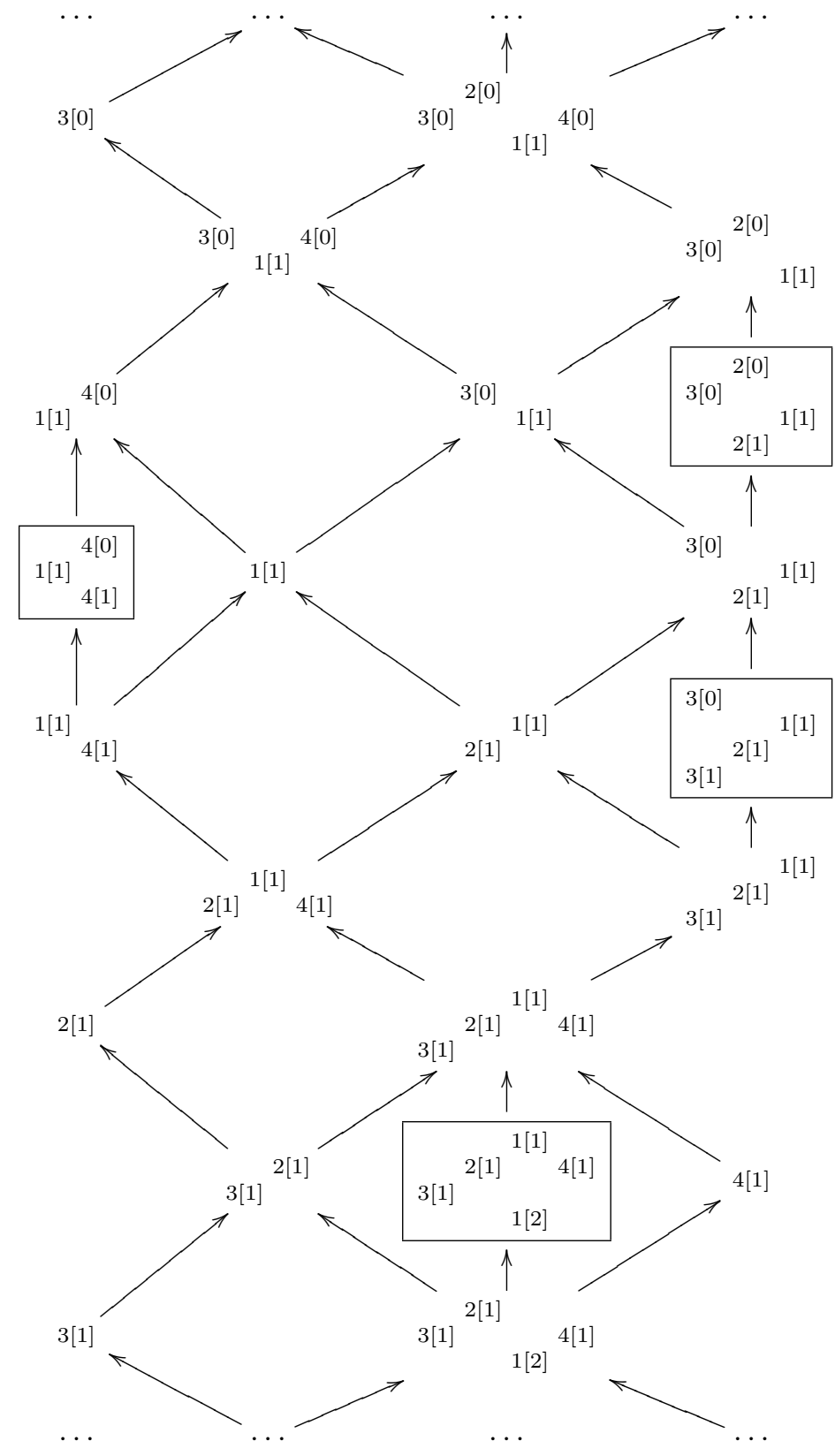

where representations are written as their composition series (each $i[r]$ represents the simple representation associated to the corresponding vertex), and projectiveinjective representations are written inside boxes. 
Let $\psi$ denote a fixed embedding of the Auslander-Reiten quiver of $(\bmod \widehat{A})^{\text {op }}$ as a full subquiver of $\widehat{\Gamma}$.

Lemma 3.6. Let $\Sigma:=\psi(\operatorname{proj} \widehat{A})$. Then there is an algebra isomorphism

$$
e_{\Sigma} \Lambda e_{\Sigma} \cong \widehat{A} \text {. }
$$

Proof. The category $(\bmod \widehat{A})^{\text {op }}$ is equivalent to the category generated by its Auslander-Reiten quiver, together with the mesh relations. Let $\Lambda^{\prime}$ be the path algebra of this Auslander-Reiten quiver quotiented by the mesh relations. Then $\Lambda^{\prime}$ is isomorphic to the endomorphism algebra of the direct sum of all finite-dimensional indecomposable modules over $\widehat{A}$. Thus

$$
e \Lambda^{\prime} e \cong \operatorname{End}_{\widehat{A}}\left(\bigoplus_{P \text { projective }} P\right)^{\mathrm{op}} \cong \widehat{A} .
$$

The quivers of $\Lambda$ and $\Lambda^{\prime}$ are the same; their relations differ only by some signs. Consider the following vertex-preserving automorphism of $\Lambda$ :

$$
\begin{aligned}
a_{(i, n+1)} & \mapsto a_{(i, n+1)} ; \\
b_{(i, n)} & \mapsto b_{(i, n)} ; \\
(c, n) & \mapsto \begin{cases}(c, n) & \text { if } n \equiv 0,1 \bmod 4 ; \\
-(c, n) & \text { else; }\end{cases} \\
(\bar{c}, n+1) & \mapsto \begin{cases}(\bar{c}, n+1) & \text { if } n \equiv 0,1 \bmod 4 ; \\
-(\bar{c}, n+1) & \text { else; }\end{cases}
\end{aligned}
$$

for any $(i, n) \in \widehat{Q}_{0}$. Under this automorphism, the relations of $\Lambda$ become the mesh relations; thus $\Lambda$ and $\Lambda^{\prime}$ are isomorphic, and this isomorphism preserves the idempotents associated to the vertices. So we have $e \Lambda e \cong e \Lambda^{\prime} e \cong \widehat{A}$.

Remark 3.7. The isomorphism $\Lambda \cong \Lambda^{\prime}$ defined above induces a $G_{V}$-equivariant isomorphism of varieties $\operatorname{rep}_{\mathbf{d}}(\Lambda) \cong \operatorname{rep}_{\mathbf{d}}\left(\Lambda^{\prime}\right)$, for any dimension vector $\mathbf{d}$. Thus we will often identify the representations of $\Lambda$ with those of $\Lambda^{\prime}$ and conversely.

Corollary 3.8. Let $\mathbf{d}$ be a finite dimension vector supported on $\psi(\operatorname{proj} \widehat{A})$, and let $W^{\mathbf{d}}$ be the corresponding graded space defined as in (3). There is a $G_{W}$-equivariant isomorphism of varieties

$$
\Psi: \mathfrak{M}_{0}^{\bullet}\left(W^{\mathbf{d}}\right) \rightarrow \operatorname{rep}_{\mathbf{d}} \widehat{A} .
$$

Proof. This follows from Lemma 3.6 and Theorem 2.4. 


\section{§3.2. The Grothendieck group for repetitive algebras}

Let $\mathcal{A}$ be an essentially small abelian category. Its split Grothendieck group $K_{0}^{\text {split }}(\mathcal{A})$ is the free abelian group generated by isomorphism classes of objects in $\mathcal{A}$, subject to the relations

$$
[X]-[X \oplus Z]+[Z]=0 \quad \text { for all objects } X \text { and } Z \text { of } \mathcal{A} .
$$

The Grothendieck group of $\mathcal{A}$ is the abelian group $K_{0}(\mathcal{A})$ obtained by adding the relations

$$
[X]-[Y]+[Z]=0 \quad \text { for all exact sequences } 0 \rightarrow X \rightarrow Y \rightarrow Z \rightarrow 0 .
$$

Let $\phi: K_{0}^{\text {split }}(\mathcal{A}) \rightarrow K_{0}(\mathcal{A})$ be the canonical surjection. In [3], M. Auslander gave a basis of Ker $\phi$ in the case when $\mathcal{A}$ is the category of finite-dimensional modules over a finite-dimensional $k$-algebra $A$ that is representation finite. We will give a similar description of Ker $\phi$ for the module category of a repetitive algebra of Dynkin type. Although the proof follows the lines of [3], we include it here for completeness.

Let $\widehat{A}$ be the repetitive algebra of the path algebra of a quiver of Dynkin type. For any vertex $i$ of the Gabriel quiver $Q^{\text {repet }}$ of $\widehat{A}$, let $\widehat{S}_{i}$ be the corresponding simple $\widehat{A}$-module, and let $\widehat{P}_{i}$ be its projective cover.

By [12, Lemma 2.5], the category mod $\widehat{A}$ has almost split sequences. For any non-projective indecomposable module $M$ in $\bmod \widehat{A}$, there exists an almost split sequence (unique up to isomorphism)

$$
0 \rightarrow \tau M \rightarrow E_{M} \rightarrow M \rightarrow 0 .
$$

For any indecomposable module $M$, let $r_{M}$ be the element of $K_{0}^{\text {split }}(\bmod \widehat{A}) \operatorname{defined}$ by

$$
r_{M}= \begin{cases}{[M]-\left[E_{M}\right]+[\tau M]} & \text { if } M \text { is not projective } \\ {[M]-[\operatorname{rad} M]} & \text { if } M \text { is projective. }\end{cases}
$$

Define a bilinear form $h$ on $K_{0}^{\text {split }}(\bmod \widehat{A})$ by

$$
h([M],[N])=\operatorname{dim}_{\mathbb{C}} \operatorname{Hom}_{\widehat{A}}(M, N) \quad \text { for any modules } M \text { and } N .
$$

We gather in the next lemma the properties of $h$ that we will need.

Lemma 3.9. (i) For any indecomposable module $M$, we have $h\left([M], r_{M}\right)=1$. (ii) If $M$ and $N$ are indecomposable and non-isomorphic, then $h\left([M], r_{N}\right)=0$. 
(iii) For any $x \in K_{0}^{\mathrm{split}}(\bmod \widehat{A})$, we have

$$
x=\sum_{M \text { indec. }} h\left(x, r_{M}\right)[M] .
$$

(iv) If $x$ and $y$ are elements of $K_{0}^{\text {split }}(\bmod \widehat{A})$ such that, for any indecomposable module $L$, we have $h(x,[L])=h(y,[L])$, then $x=y$. The same holds if $h([L], x)=h([L], y)$ for any indecomposable $L$.

Proof. We first prove statement (i). Assume that $M$ is indecomposable and is not projective. Then there is an exact sequence

$$
0 \rightarrow \operatorname{Hom}_{\widehat{A}}(M, \tau M) \rightarrow \operatorname{Hom}_{\widehat{A}}\left(M, E_{M}\right) \rightarrow \operatorname{Hom}_{\widehat{A}}(M, M) \rightarrow S(M) \rightarrow 0
$$

and $h\left([M], r_{M}\right)=\operatorname{dim} S(M)$. Notice that $S(M)$ is isomorphic to the only simple $\operatorname{End}_{\widehat{A}}(M)$-module, which is one-dimensional. Now, if $M$ is projective, then the equality $h\left([M], r_{M}\right)=1$ follows from the fact that $M / \operatorname{rad} M$ is a one-dimensional simple module.

Statement (ii) follows from the definition of almost split sequences; indeed, if $M$ and $N$ are indecomposable and non-isomorphic, with $N$ non-projective, then we have an exact sequence

$$
0 \rightarrow \operatorname{Hom}_{\widehat{A}}(M, \tau N) \rightarrow \operatorname{Hom}_{\widehat{A}}\left(M, E_{N}\right) \rightarrow \operatorname{Hom}_{\widehat{A}}(M, N) \rightarrow 0
$$

If $N$ is projective, then any morphism from $M$ to $N$ has its image contained in $\operatorname{rad} N$, so that $\operatorname{Hom}_{\widehat{A}}(M, N)=\operatorname{Hom}_{\widehat{A}}(M, \operatorname{rad} N)$. The result follows.

The equation of (iii) follows directly from (i) and (ii). To prove (iv), notice that if $h(x,[L])=0$ for any indecomposable $L$, then $x=0$ by (iii). Thus, if $h(x,[L])=h(y,[L])$ for any indecomposable $L$, then $x-y$ has to be zero. The second equality is obtained by applying the first to the opposite algebra $\widehat{A}^{\text {op }}$ and applying the duality functor.

Proposition 3.10. Let $A=\mathbb{C} Q$ for a quiver $Q$ of Dynkin type. Let $\widehat{A}$ be the repetitive algebra of $A$.

(i) For any $x \in K_{0}^{\mathrm{split}}(\bmod \widehat{A})$, we have

$$
x=\sum_{M \text { indec. }} h([M], x) r_{M} .
$$

(ii) $\left\{r_{M} \mid M\right.$ is indecomposable $\}$ is a basis for $K_{0}^{\mathrm{split}}(\bmod \widehat{A})$.

(iii) $\left\{r_{M} \mid M\right.$ is indecomposable and non-projective $\}$ is a basis for Ker $\phi$. 
Proof. To prove (i), notice first that the sum is finite. This follows from the fact that for a given $N$, there is a finite number of indecomposable modules $M$ such that $h([M],[N])$ is non-zero. Thus the sum in (i) is an element of $K_{0}^{\text {split }}(\bmod \widehat{A})$. Next, for any indecomposable module $L$, we have

$$
\begin{aligned}
h\left([L], \sum_{M \text { indec. }} h([M], x) r_{M}\right) & =\sum_{M \text { indec. }} h([M], x) h\left([L], r_{M}\right) \\
& =h([L], x) h\left([L], r_{L}\right)=h([L], x) .
\end{aligned}
$$

Here, the second equality follows from Lemma 3.9(ii). Applying Lemma 3.9(iv), we deduce (i).

It follows from (i) that the set $\left\{r_{M} \mid M\right.$ is indecomposable $\}$ generates the group $K_{0}^{\text {split }}(\bmod \widehat{A})$. The fact that its elements are linearly independent follows from Lemma 3.9(i), (ii): if $\sum_{M \text { indec. }} \lambda_{M} r_{M}=0$, then applying $h([L], ?)$ yields the equality $\lambda_{L}=0$, for any indecomposable $L$. This proves (ii).

To prove (iii), notice that $\phi\left(r_{M}\right)=0$ for any non-projective indecomposable $M$, and that $\phi\left(r_{\widehat{P}_{i}}\right)=\left[\widehat{S}_{i}\right]$. Thus no non-trivial linear combination of the $r_{\widehat{P}_{i}}$ lies in the kernel of $\phi$. This finishes the proof.

Corollary 3.11. Let $N$ be a representation of $\widehat{A}$ of dimension vector $\mathbf{d}$. Then in $K_{0}^{\text {split }}(\bmod \widehat{A})$ we have

$$
-[N]+\sum_{i} d_{i}\left[\widehat{S}_{i}\right]=\sum_{M \text { non-proj. }} \operatorname{dim}(\operatorname{proj}(\Omega M, N)) r_{M},
$$

where $\operatorname{proj}(A, B)$ is the space of morphisms from $A$ to $B$ that factor through a projective representation, and where $\Omega$ is the syzygy. In particular, $N$ is completely determined up to isomorphism by the values of $\operatorname{dim}(\operatorname{proj}(M, N))$, as $M$ ranges over all non-projective indecomposables.

Proof. The element $-[N]+\sum_{i} d_{i}\left[\widehat{S}_{i}\right]$ lies in the kernel of $\phi$. Thus, by Proposition 3.10 , it can be written in a unique way as a linear combination of some $r_{M}$, for $M$ indecomposable non-projective, and the coefficient of $r_{M}$ is

$$
h\left([M],-[N]+\sum_{i} d_{i}\left[\widehat{S}_{i}\right]\right) .
$$

Now let $P^{M}$ be a minimal projective cover of $M$, so that we have a short exact sequence

$$
0 \rightarrow \Omega M \rightarrow P^{M} \rightarrow M \rightarrow 0
$$

and an exact sequence

$$
0 \rightarrow \operatorname{Hom}(M, N) \rightarrow \operatorname{Hom}\left(P^{M}, N\right) \rightarrow \operatorname{Hom}(\Omega M, N) .
$$


From this we see that $\operatorname{proj}(\Omega M, N) \cong \operatorname{Hom}\left(P^{M}, N\right) / \operatorname{Hom}(M, N)$. Moreover, since $\operatorname{Hom}\left(P^{M}, N\right)$ only depends on the dimension vector of $N$, it is isomorphic to $\operatorname{Hom}\left(P^{M}, \bigoplus_{i} \widehat{S}_{i}^{d_{i}}\right)$, which in turn is isomorphic to $\operatorname{Hom}\left(M, \bigoplus_{i} \widehat{S}_{i}^{d_{i}}\right)$, since $P^{M}$ is a minimal projective cover of $M$. Thus

$$
\begin{aligned}
\operatorname{dim} \operatorname{proj}(\Omega M, N) & =\operatorname{dim} \operatorname{Hom}\left(P^{M}, N\right)-\operatorname{dim} \operatorname{Hom}(M, N) \\
& =\operatorname{dim} \operatorname{Hom}\left(M, \bigoplus_{i} \widehat{S}_{i}^{d_{i}}\right)-\operatorname{dim} \operatorname{Hom}(M, N) \\
& =h\left([M],-[N]+\sum_{i} d_{i}\left[\widehat{S}_{i}\right]\right) .
\end{aligned}
$$

This proves the first claim. Since the $r_{M}$ form a basis of the kernel of $\phi$, a module $N^{\prime}$ not isomorphic to $N$ will give rise to different values for the $h\left([M],\left[N^{\prime}\right]\right)$. This proves the second claim.

\section{§3.3. A bijection between strata and orbits}

Recall the algebra

$$
\Lambda^{\prime}=\operatorname{End}_{\widehat{A}}\left(\bigoplus_{M \text { indec. }} M\right)^{\mathrm{op}}
$$

where the sum is taken over all isomorphism classes $M$ of indecomposable representations of $\widehat{A}$.

Lemma 3.12. Let $\mathbf{d}$ be a finite dimension vector supported on $\psi(\operatorname{proj} \widehat{A})$. Let $N$ be a representation of $\widehat{A}$ of dimension $\mathbf{d}$. Consider the left $\Lambda^{\prime}$-module

$$
\underline{N}=\operatorname{proj}\left(\bigoplus_{M \text { indec. }} M, N\right) .
$$

Then $\underline{N}$, regarded as a point of $\Lambda^{\bullet}\left(V, W^{\mathbf{d}}\right)$ for some $V$, is stable and dominant.

Proof. To prove that $\underline{N}$ is stable, notice that if $L$ is an indecomposable nonprojective representation of $\widehat{A}$ and if $f \in V_{\psi(L)}=\operatorname{proj}(L, N)$ is non-zero, then any projective cover $g: P \rightarrow L$ is such that the composition $f \circ g$ does not vanish, since $g$ is surjective. Thus $g$, viewed as an element of $\Lambda^{\prime}$, sends $f$ to a non-zero element of $W_{\psi(P)}=\operatorname{proj}(P, N)$. Therefore the subrepresentation of $\underline{N}$ generated by $f$ is not supported on $V$.

To prove that $\underline{N}$ is dominant, we must prove the inequality

$$
\operatorname{dim} W_{(i, n)}-\operatorname{dim} V_{(i, n+1)}-\operatorname{dim} V_{(i, n-1)}+\sum_{j \sim i} \operatorname{dim} V_{(j, n)} \geq 0
$$


for all $(i, n) \in \widehat{\Gamma}_{0} \backslash \widehat{Q}_{0}$. All these spaces lie in a "mesh" of the form

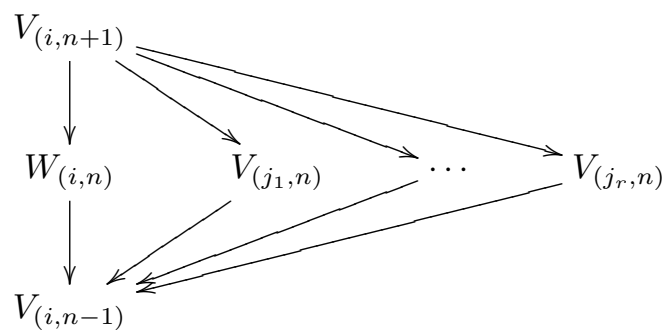

whose preimage under $\psi$ corresponds to an almost split sequence

$$
0 \rightarrow \tau M \rightarrow \bigoplus_{k} E_{k} \rightarrow M \rightarrow 0 .
$$

Notice that the middle term has a projective summand $P$ if, and only if, $(i, n)=$ $\psi(P)$. Thus, since $V_{\psi(L)}=\operatorname{proj}(L, N)$, the above inequality becomes

$$
-\operatorname{dim} \operatorname{proj}(M, N)-\operatorname{dim} \operatorname{proj}(\tau M, N)+\sum_{k} \operatorname{dim} \operatorname{proj}\left(E_{k}, N\right) \geq 0,
$$

where the counterpart of $\operatorname{dim} W_{(i, n)}$ is included in the sum and corresponds to the possible projective summand $P$. Since projectives are injectives, if a morphism $\tau M \rightarrow N$ factors through a projective, then it factors through $E=\bigoplus_{k} E_{k}$. Thus we get a sequence

$$
\operatorname{proj}(M, N) \rightarrow \operatorname{proj}(E, N) \rightarrow \operatorname{proj}(\tau M, N),
$$

which is not exact, but whose first map is injective and second map is surjective, and such that their composition vanishes. Thus

$$
\operatorname{dim} \operatorname{proj}(E, N) \geq \operatorname{dim} \operatorname{proj}(M, N)+\operatorname{proj}(\tau M, N),
$$

and $N$ is dominant.

Remark 3.13. One can show that the $G_{V}$-orbit of $\underline{N}$ is always closed. This result, in a more general context, is proved in a work of Bernhard Keller and Sarah Scherotzke [13]. We are grateful to them for kindly explaining their result to us prior to its appearance on the arXiv.

Theorem 3.14. Let $\mathbf{d}$ be a finite dimension vector supported on $\psi(\operatorname{proj} \widehat{A})$. Then the isomorphism

$$
\Psi: \mathfrak{M}_{0}^{\bullet}\left(W^{\mathbf{d}}\right) \rightarrow \operatorname{rep}_{\mathbf{d}}(\widehat{A})
$$


of Corollary 3.8 induces a bijection between the following sets:

(i) the set of isomorphism classes of representations of $\widehat{A}$ with dimension vector $\mathbf{d}$;

(ii) the set of dominant pairs $\left(V, W^{\mathbf{d}}\right)$;

(iii) the set of non-empty strata $\mathfrak{M}_{0}^{\bullet \text { reg }}\left(V, W^{\mathbf{d}}\right)$.

Proof. Lemma 3.12 gives a map from set (i) to set (ii), sending a representation $N$ to the dominant pair $\left(V, W^{\mathbf{d}}\right)$ associated to $\underline{N}$. This map is injective since, by Corollary 3.11, $N$ is completely determined by the values of $\operatorname{dim}(\operatorname{proj}(L, N))$ for all non-projective indecomposable $\widehat{A}$-modules $L$, and these give the graded dimension of $V$.

Assume now that $\left(V, W^{\mathbf{d}}\right)$ is a dominant pair. Consider the element

$$
\sum_{i} d_{i}\left[\widehat{S}_{i}\right]-\sum_{M} \operatorname{dim} V_{\psi(\Omega M)} r_{M} \in K_{0}^{\mathrm{split}}(\widehat{A}) .
$$

If $L$ is an indecomposable non-projective representation, then the coefficient of $[L]$ in this element is given by

$$
\sum_{i} d_{i} \delta_{\widehat{S}_{i}, L}-\operatorname{dim} V_{\psi(\Omega L)}-\operatorname{dim} V_{\psi\left(\Omega \tau^{-1} L\right)}+\sum_{\Omega L \rightarrow N} \operatorname{dim} V_{\psi(N)}
$$

This corresponds to the almost split sequence ending in $\Omega \tau^{-1} L$. (Note that if $L$ is simple, then $\Omega L$ is the radical of a projective module, and the corresponding projective appears in the middle of this almost split sequence). Since $\left(V, W^{\mathbf{d}}\right)$ is dominant, this quantity is non-negative. On the other hand, if $P$ is an indecomposable projective, then the coefficient of $[P]$ is non-negative, since it appears only with non-negative coefficients in all the elements $r_{M}$.

Therefore $\sum_{i} d_{i}\left[\widehat{S}_{i}\right]-\sum_{M}\left(\operatorname{dim} V_{\psi \Omega M}\right) r_{M}=[N]$ for some representation $N$ of $\widehat{A}$ with dimension vector $\mathbf{d}$. This defines an injective map from set (ii) to set (i). Since they are finite sets, they are in bijection. Note that the two maps that we defined are inverse to each other.

Now, we know from Lemma 3.12 that any representation $N$ gives rise to a representation $\underline{N}$ of $\Lambda^{\prime}$ that is both stable and dominant. Therefore the corresponding stratum $\mathfrak{M}_{0}^{\bullet r e g}\left(V, W^{\mathbf{d}}\right)$ is non-empty. This gives an injective map from set (i) to set (iii). Finally, if $\mathfrak{M}_{0}^{\bullet r e g}\left(V, W^{\mathbf{d}}\right)$ is non-empty, then $\left(V, W^{\mathbf{d}}\right)$ is dominant; this gives an injective map from set (iii) to set (ii), and we have a bijection.

The proof of Theorem 3.14 gives us a description of the space $V$ in the dominant pair $(V, W)$ associated to a representation $N$ of $\widehat{A}$. 
Corollary 3.15. Let $N$ be a representation of $\widehat{A}$ of dimension vector $\mathbf{d}$, and let $\mathfrak{M}_{0}^{\text {•reg }}\left(V, W^{\mathbf{d}}\right)$ be the corresponding non-empty stratum. Then the following integers are equal for any indecomposable non-projective $\widehat{A}$-module $M$ :

(i) $\operatorname{dim} V_{\psi M}$;

(ii) $\operatorname{dim} \operatorname{proj}(M, N)$;

(iii) $\operatorname{dim} \operatorname{Hom}_{\widehat{A}}\left(\Omega^{-1} M, \bigoplus_{i} \widehat{S}_{i}^{d_{i}}\right)-\operatorname{dim} \operatorname{Hom}_{\widehat{A}}\left(\Omega^{-1} M, N\right)$;

(iv) the coefficient of $r_{\Omega^{-1} M}$ in the equality

$$
-[N]+\sum_{i} d_{i}\left[\widehat{S}_{i}\right]=\sum_{M \text { non-proj. }} \lambda_{M} r_{M} .
$$

Proof. The equality of (i) and (ii) follows from the bijection given in Theorem 3.14 and from the definition of $\underline{N}$. The equality of (ii) and (iii) was given at the end of the proof of Corollary 3.11. The equality of (ii) and (iv) follows from Corollary 3.11 .

Remark 3.16. We are grateful to Christof Geiss for sharing with us his observation of the equality of (i) and (iii) in Corollary 3.15. Note that by Nakajima's theory, the stratum $\mathfrak{M}_{0}^{\bullet r e g}\left(V, W^{\mathbf{d}}\right)$ contains the stratum $\mathfrak{M}_{0}^{\text {reg }}\left(V^{\prime}, W^{\mathbf{d}}\right)$ in its closure if and only if $\operatorname{dim} V_{(i, n)} \geq \operatorname{dim} V_{(i, n)}^{\prime}$ for every $(i, n) \in \widehat{Q}_{0}$. Thus, using the equality of (i) and (iii), we find that a d-dimensional $\widehat{A}$-module $N^{\prime}$ lies in the closure of the orbit of $N$ if and only if $\operatorname{dim}_{\operatorname{Hom}_{\widehat{A}}}(M, N) \leq \operatorname{dim}_{\widehat{A}} \operatorname{Hom}_{\widehat{A}}\left(M, N^{\prime}\right)$ for every indecomposable $\widehat{A}$-module $M$. Hence we recover that for the repetitive algebra of a Dynkin quiver, which is locally of finite representation type, the degeneration order and the Hom order coincide [23].

Remark 3.17. In case $W$ is supported on the image by $\psi$ of projectives associated to vertices $1[r], 2[r], \ldots, n[r]$ for some integer $r$, Theorem 3.14 recovers Theorem 9.11 of [11]. In that paper, the place of the $W$ 's corresponds to the place of the simple modules in $\bmod \mathbb{C} Q$. Our place for the $W$ 's is the place of the projective indecomposable modules in mod $\widehat{Q}$. To see that the two choices give the same pattern for the placing of the $W$ 's, notice that the syzygy $\Omega$ (which is an autoequivalence of the triangulated category $\bmod \widehat{A}$ ) sends any simple $S$ to the $\operatorname{radical} \operatorname{rad} P$ of a projective module $P$, and that $\operatorname{rad} P$ is the start of an almost split sequence having $P$ in its middle term.

Proposition 3.18. Let $N$ be a representation of $\widehat{A}$ of dimension vector $\mathbf{d}$, and write

$$
N=\bigoplus_{M \text { indec. }} M^{a_{M}}
$$


Then, if $M$ is not projective, we have

$$
a_{\Omega^{-1} \tau M}=-\operatorname{dim} \operatorname{proj}(M, N)-\operatorname{dim} \operatorname{proj}(\tau M, N)+\sum_{k} \operatorname{dim} \operatorname{proj}\left(E_{k}, N\right),
$$

where $0 \rightarrow \tau M \rightarrow \bigoplus_{k} E_{k} \rightarrow M \rightarrow 0$ is an almost split sequence.

Proof. Three cases arise:

- the almost split sequences ending in $M$ and in $\Omega^{-1} M$ have no projective summands in their middle terms;

- the almost split sequence ending in $M$ has a projective summand $\widehat{P}$ (this is equivalent to $M$ being isomorphic to $\widehat{P} / \operatorname{soc}(\widehat{P}))$;

- the almost split sequence ending in $\Omega^{-1} M$ has a projective summand $\widehat{Q}$ (this is equivalent to $\Omega^{-1} M$ being isomorphic to $\left.\widehat{Q} / \operatorname{soc}(\widehat{Q})\right)$.

Let $\delta$ (resp. $\epsilon$ ) be equal to 1 if $M\left(\right.$ resp. $\left.\Omega^{-1} M\right)$ is isomorphic to $\widehat{P} / \operatorname{soc}(\widehat{P})($ resp. $\widehat{Q} / \operatorname{soc}(\widehat{Q}))$, and 0 otherwise. We can therefore write the almost split sequences as

$$
0 \rightarrow \tau M \rightarrow \widehat{P}^{\delta} \oplus \bigoplus_{E_{k} \text { non-proj. }} E_{k} \rightarrow M \rightarrow 0
$$

resp.

$$
0 \rightarrow \Omega^{-1} \tau M \rightarrow \widehat{Q}^{\epsilon} \oplus \bigoplus_{E_{k} \text { non-proj. }} \Omega^{-1} E_{k} \rightarrow \Omega^{-1} M \rightarrow 0
$$

Then, using Corollary 3.15, we get

$$
\begin{aligned}
& -\operatorname{dim} \operatorname{proj}(M, N)-\operatorname{dim} \operatorname{proj}(\tau M, N)+\sum_{k} \operatorname{dim} \operatorname{proj}\left(E_{k}, N\right) \\
& =-\operatorname{dim} \operatorname{proj}(M \oplus \tau M, N)+\sum_{E_{k} \text { non-proj. }} \operatorname{dim} \operatorname{proj}\left(E_{k}, N\right)+\delta \operatorname{dim} \operatorname{proj}(\widehat{P}, N) \\
& =-\operatorname{dim} \operatorname{proj}(M \oplus \tau M, N)+\sum_{E_{k} \text { non-proj. }} \operatorname{dim} \operatorname{proj}\left(E_{k}, N\right)+\delta d_{\psi(\widehat{P})} \\
& =h\left(-r_{\Omega^{-1} M}-\epsilon[\widehat{Q}], \sum_{i} d_{i} \widehat{S}_{i}-[N]\right)+\delta d_{\psi(\widehat{P})} \\
& =a_{\Omega^{-1} \tau M}-\sum_{i} d_{i} \delta_{\Omega^{-1} \tau M, \widehat{S}_{i}}+\delta d_{\psi(\widehat{P})}=a_{\Omega^{-1} \tau M}-\delta d_{\psi(\widehat{P})}+\delta d_{\psi(\widehat{P})}=a_{\Omega^{-1} \tau M} .
\end{aligned}
$$

Here we have used the fact that, since $\widehat{Q}$ is projective-injective,

$$
h\left([\widehat{Q}], \sum_{i} d_{i} \widehat{S}_{i}-[N]\right)=0 .
$$

Example 3.19. We continue Example 3.5. Put $W_{(3,8)}=W_{(1,4)}=W_{(3,0)}=\mathbb{C}$; these spaces correspond to the images of some projectives under some inclusion $\psi$ of $\bmod \widehat{A}$ into $\widehat{\Gamma}$, so that Theorem 3.14 applies. 


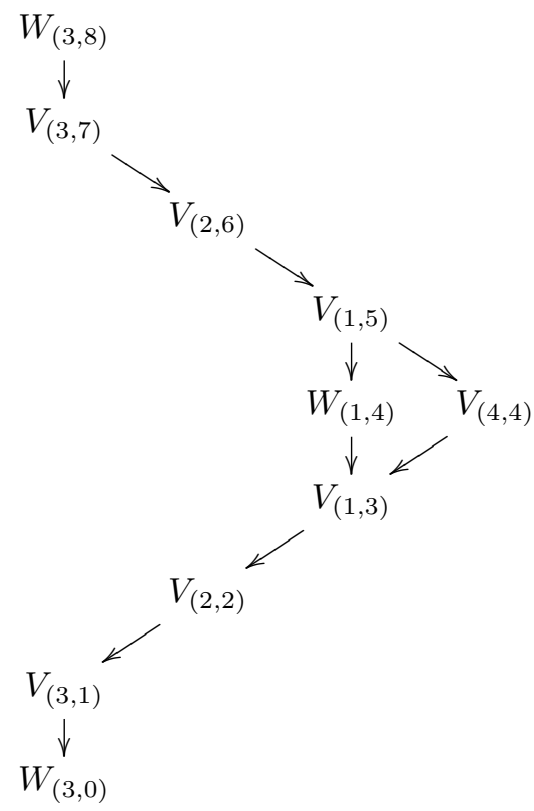

There are four isomorphism classes of representations of $\widehat{A}$ of the corresponding dimension vector, which is supported on the following subquiver of $Q^{\text {repet: }}$

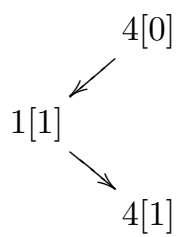

Corollary 3.15 gives the dominant pair $(V, W)$ associated to each isomorphism class. The table below describes the bijection explicitly.

\begin{tabular}{lccccc}
\hline & $4[0] \oplus 1[1] \oplus 4[1]$ & ${ }^{4[1]}{ }^{4[0]} \oplus 4[1]$ & $4[0]$ & ${ }^{1[1]}{ }_{4[1]}$ & ${ }^{1[1]}{ }^{4[0]}$ \\
\hline $\operatorname{dim} V_{(3,7)}$ & 0 & 1 & 0 & 1 \\
$\operatorname{dim} V_{(2,6)}$ & 0 & 1 & 0 & 1 \\
$\operatorname{dim} V_{(1,5)}$ & 0 & 1 & 0 & 1 \\
$\operatorname{dim} V_{(4,4)}$ & 0 & 0 & 0 & 1 \\
$\operatorname{dim} V_{(1,3)}$ & 0 & 0 & 1 & 1 \\
$\operatorname{dim} V_{(2,2)}$ & 0 & 0 & 1 & 1 \\
$\operatorname{dim} V_{(3,1)}$ & 0 & 0 & 1 & 1 \\
\hline
\end{tabular}


Example 3.20. The bijection of Theorem 3.14 does not hold for a general $W$. Indeed, let $Q$ be the quiver $1 \rightarrow 2$ of type $\mathbb{A}_{2}$. Put $W_{(1,3)}=W_{(1,1)}=W_{(2,0)}=\mathbb{C}$.

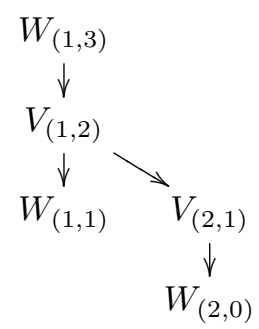

Then Theorem 2.4 gives an isomorphism between $\mathfrak{M}_{0}^{\bullet \text { reg }}(W)$ and the variety of representations of dimension vector $(1,1,1)$ of the quiver

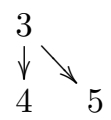

Then there are three dominant pairs for $W^{\mathbf{d}}$, given by

$$
\left(\operatorname{dim} V_{(1,2)}, \operatorname{dim} V_{(2,1)}\right) \in\{(0,0),(1,0),(1,1)\},
$$

while there are four orbits for this dimension vector, namely those of

$$
3 \oplus 4 \oplus 5, \quad{ }_{4}^{3} \oplus 5, \quad 3_{5} \oplus 4, \quad 3_{4}^{3} .
$$

\section{§4. Composition multiplicities for quantum loop algebras}

\section{§4.1. Quantum loop algebras and $q$-characters}

Let $\mathfrak{g}$ be a complex simple Lie algebra of type $\mathbb{A}, \mathbb{D}$ or $\mathbb{E}$. Let $Q$ be an arbitrary orientation of the Dynkin diagram of $\mathfrak{g}$, that is, a Dynkin quiver of the same type as $\mathfrak{g}$. Let $L \mathfrak{g}$ be the loop algebra attached to $\mathfrak{g}$, and let $U_{q}(L \mathfrak{g})$ be the associated quantum enveloping algebra. We assume that the deformation parameter $q \in \mathbb{C}^{*}$ is not a root of unity.

By [6], every finite-dimensional $U_{q}(L \mathfrak{g})$-module $M$ (of type 1 ) has a $q$-character $\chi_{q}(M)$, which is a Laurent polynomial in

$$
\mathcal{Y}:=\mathbb{Z}\left[Y_{i, a}^{ \pm 1} \mid i \in Q_{0}, a \in \mathbb{C}^{*}\right]
$$

encoding the dimensions of its loop weight spaces. These $q$-characters generate a commutative subalgebra of $\mathcal{Y}$ isomorphic to the Grothendieck ring of the category of finite-dimensional irreducible $U_{q}(L \mathfrak{g})$-modules. In other words, finitedimensional simple $U_{q}(L \mathfrak{g})$-modules are characterized by their $q$-characters up to isomorphism. 
As in many other representation theories, the $q$-characters of simple modules do not have a simple description and they are calculated via the $q$-characters of more accessible modules called standard modules. As in [11], we will label simple modules $L(m)$ and standard modules $M(m)$ by the unique monomial $m$ of their $q$-characters corresponding to their highest weight vectors. These highest weight monomials $m$ are called dominant because they only involve nonnegative powers of the variables $Y_{i, a}$.

Hence, a basic question is to calculate, for every pair $\left(m, m^{\prime}\right)$ of dominant monomials, the composition multiplicities $\zeta_{m, m^{\prime}}$ appearing in the expansions

$$
\chi_{q}\left(M\left(m^{\prime}\right)\right)=\sum_{m} \zeta_{m, m^{\prime}} \chi_{q}(L(m))
$$

\section{$\S 4.2$. Nakajima varieties and composition multiplicities}

By a classical reduction procedure (see e.g. [10]), it is enough to determine the multiplicities $\zeta_{m, m^{\prime}}$ corresponding to pairs $\left(m, m^{\prime}\right)$ of dominant monomials in the variables of the set

$$
\mathcal{Z}:=\left\{Y_{i, q^{n}} \mid(i, n) \in \widehat{\Gamma}_{0} \backslash \widehat{Q}_{0}\right\}
$$

where $\widehat{\Gamma}_{0}$ and $\widehat{Q}_{0}$ have been defined in $\S 2.1$. The modules $L(m)$ where $m$ ranges over all dominant monomials in the variables of $\mathcal{Z}$ generate a tensor subcategory $\mathcal{C}_{\mathbb{Z}}$ of the category of finite-dimensional $U_{q}(L \mathfrak{g})$-modules.

From now on, we shall only consider monomials in the variables of $\mathcal{Z}$, and write for short $Y_{i, n}$ instead of $Y_{i, q^{n}}$. In particular, we define for $(i, n) \in \widehat{Q}_{0}$ the Laurent monomial

$$
A_{i, n}:=Y_{i, n-1} Y_{i, n+1} \prod_{j \sim i} Y_{j, n}^{-1}
$$

Given a finite dimension vector $\mathbf{d}$ on $\widehat{\Gamma}$ and the two corresponding graded vector spaces $V$ and $W$ as in (1), we define two monomials

$$
A^{V}:=\prod_{(i, n) \in \widehat{Q}_{0}} A_{i, n}^{-d_{i, n}}, \quad Y^{W}:=\prod_{(i, n) \in \widehat{\Gamma}_{0} \backslash \widehat{Q}_{0}} Y_{i, n}^{d_{i, n}} .
$$

It is easy to check that the pair $(V, W)$ is dominant in the sense of $\S 2.2$ if and only if the monomial $m_{\mathbf{d}}:=Y^{W} A^{V}$ is dominant, that is, every variable $Y_{i, n}$ has a non-negative exponent in $m_{\mathbf{d}}$.

Given a dominant pair $(V, W)$ such that $\mathfrak{M}_{0}^{\bullet \text { reg }}(V, W) \neq \emptyset$, denote by $I C_{W}(V)$ the intersection cohomology complex of the closure of the stratum $\mathfrak{M}_{0}^{\bullet}{ }^{\text {reg }}(V, W)$. Let $\mathcal{H}^{i}\left(I C_{W}(V)\right)$ be its $i$ th cohomology sheaf, and $\mathcal{H}^{i}\left(I C_{W}(V)\right)_{V^{\prime}}$ be the stalk of this sheaf at a point of $\mathfrak{M}_{0}^{\bullet \text { reg }}\left(V^{\prime}, W\right)$. We can now state one of the main results of Nakajima's geometric approach to the representation theory of $U_{q}(L \mathfrak{g})$. 
Theorem $4.1([18, \S 8])$. Let $m$ and $m^{\prime}$ be two dominant monomials in the variables of $\mathcal{Z}$. The multiplicity of the simple module $L(m)$ as a composition factor of the standard module $M\left(m^{\prime}\right)$ is given by

$$
\zeta_{m, m^{\prime}}=\sum_{i \geq 0} \operatorname{dim} \mathcal{H}^{i}\left(I C_{W}(V)\right)_{V^{\prime}}
$$

for any pair of strata $\mathfrak{M}_{0}^{\bullet \text { reg }}(V, W)$ and $\mathfrak{M}_{0}^{\bullet \text { reg }}\left(V^{\prime}, W\right)$ such that $m=Y^{W} A^{V}$ and $m^{\prime}=Y^{W} A^{V^{\prime}}$.

\section{$\S 4.3$. Parametrization of irreducible modules}

Let $N$ be a representation of $\widehat{A}$ of dimension d. By Theorem 3.14, $N$ corresponds to a unique dominant pair $\left(V, W^{\mathbf{d}}\right)$. This allows us to attach to $N$ a dominant monomial

$$
m_{N}:=Y^{W^{\mathrm{d}}} A^{V}
$$

in the variables of $\mathcal{Z}$.

Let $N^{\prime}$ be another $\widehat{A}$-module of dimension $\mathbf{d}^{\prime}$. Suppose that $N \simeq N^{\prime} \oplus P$, where $P$ is a projective $\widehat{A}$-module. Let $\left(V^{\prime}, W^{\mathbf{d}^{\prime}}\right)$ and $\left(V^{\prime \prime}, W^{\prime \prime}\right)$ be the dominant pairs associated with $N^{\prime}$ and $P$ by Theorem 3.14. Then we have the following isomorphisms of graded vector spaces:

$$
W^{\mathrm{d}} \simeq W^{\mathbf{d}^{\prime}} \oplus W^{\prime \prime}, \quad V \simeq V^{\prime} \oplus V^{\prime \prime} .
$$

The second isomorphism follows from the fact that, by Corollary 3.15, the dimensions of the graded components of $V$ are equal to the dimensions of the spaces $\operatorname{proj}(M, N)$ for the indecomposable non-projective $\widehat{A}$-modules $M$, and these dimensions are additive with respect to $N$. Moreover, since $P$ is projective-injective,

$$
\operatorname{dim} \operatorname{proj}(M, P)=\operatorname{dim} \operatorname{Hom}_{\widehat{A}}(M, P),
$$

which is additive on the almost split sequences $\tau M \rightarrow E \rightarrow M$ of the proof of Lemma 3.12. It follows that

$$
\operatorname{dim} W_{(i, n)}^{\prime \prime}-\operatorname{dim} V_{(i, n+1)}^{\prime \prime}-\operatorname{dim} V_{(i, n-1)}^{\prime \prime}+\sum_{j \sim i} \operatorname{dim} V_{(j, n)}^{\prime \prime}=0
$$

for every $(i, n) \in \widehat{\Gamma}_{0} \backslash \widehat{Q_{0}}$. Therefore $Y^{W^{\prime \prime}} A^{V^{\prime \prime}}=1$, and

$$
m_{N}=Y^{W^{\mathrm{d}}} A^{V}=Y^{W^{\mathrm{d}^{\prime}}} A^{V^{\prime}}=m_{N^{\prime}} .
$$

Hence the monomial $m_{N}$ depends only on the isomorphism class of $N$ in the stable category $\underline{\bmod } \widehat{A}$. 
So we can assume that $N$ has no projective indecomposable summand and write

$$
N=\bigoplus_{M \text { indec. nonproj. }} M^{a_{M}}
$$

Let us write the dominant monomial $m_{N}$ as

$$
m_{N}=\prod_{(i, n) \in \widehat{\Gamma}_{0} \backslash \widehat{Q}_{0}} Y_{i, n}^{b_{i, n}}
$$

and let us express the exponents $b_{i, n}$ in terms of the multiplicities $a_{M}$. By definition of $m_{N}$, we have

$$
b_{i, n}=\operatorname{dim} W_{(i, n)}^{\mathbf{d}}-\operatorname{dim} V_{(i, n+1)}-\operatorname{dim} V_{(i, n-1)}+\sum_{j \sim i} \operatorname{dim} V_{(j, n)} .
$$

Now, arguing as in the proof of Lemma 3.12, we get

$$
b_{i, n}=-\operatorname{dim} \operatorname{proj}(M, N)-\operatorname{dim} \operatorname{proj}(\tau M, N)+\sum_{k} \operatorname{dim} \operatorname{proj}\left(E_{k}, N\right)
$$

where the almost split sequence

$$
0 \rightarrow \tau M \rightarrow \bigoplus_{k} E_{k} \rightarrow M \rightarrow 0
$$

corresponds under $\psi$ to the mesh containing the spaces $W_{i, n}^{\mathbf{d}}, V_{i, n+1}, V_{i, n-1}$, and $V_{j, n}$ for $j \sim i$. In other words, $M=\psi^{-1}(i, n+1)$. Now, applying Proposition 3.18, we get

$$
b_{i, n}=a_{\Omega^{-1} \tau \psi^{-1}(i, n+1)} .
$$

In particular we see that the indecomposable non-projective $\widehat{A}$-modules $N$ have their corresponding monomials $m_{N}$ equal to the variables $Y_{i, n}$ of $\mathcal{Z}$. Thus, summarizing the above discussion, we have proved:

Proposition 4.2. The map $N \mapsto L\left(m_{N}\right)$ induces a bijection between the isomorphism classes of objects in the stable category $\underline{\bmod } \widehat{A}$ and the simple objects of $\mathcal{C}_{\mathbb{Z}}$.

\section{§4.4. Composition multiplicities}

Let $M\left(m^{\prime}\right)$ be a standard module in the subcategory $\mathcal{C}_{\mathbb{Z}}$, and let $N^{\prime}$ be a module over the repetitive algebra $\widehat{A}$ such that $m^{\prime}=m_{N^{\prime}}$. By Proposition $4.2, N^{\prime}$ exists and its isoclass is completely determined by $m^{\prime}$, up to projective summands. Let d be the dimension vector of $N^{\prime}$. We can now state the main result of this section. 
Theorem 4.3. The multiplicities of the composition factors of $M\left(m^{\prime}\right)$ are equal to

$$
\zeta_{m_{N}, m^{\prime}}=\sum_{i \geq 0} \operatorname{dim} \mathcal{H}^{i}\left(I C\left(\mathcal{O}_{N}\right)\right)_{N^{\prime}}
$$

Here $\mathcal{O}_{N}$ denotes the orbit of a module $N$ in $\operatorname{rep}_{\mathbf{d}} \widehat{A}$ containing $N^{\prime}$ in its closure, $I C\left(\mathcal{O}_{N}\right)$ is the intersection cohomology complex of the closure of $\mathcal{O}_{N}$, and $\mathcal{H}^{i}\left(I C\left(\mathcal{O}_{N}\right)\right)_{N^{\prime}}$ is the stalk of the ith cohomology sheaf of $I C\left(\mathcal{O}_{N}\right)$ at $N^{\prime}$.

Proof. Let $\left(V^{\prime}, W^{\mathbf{d}}\right)$ be the dominant pair corresponding to $N^{\prime}$. It is well known that if $\zeta_{m, m^{\prime}} \neq 0$ we must have $m=m^{\prime} A^{V^{\prime \prime}}$ for some $\widehat{Q}_{0}$-graded space $V^{\prime \prime}$. Hence, putting $V:=V^{\prime} \oplus V^{\prime \prime}$, we have $m=Y^{W^{\mathrm{d}}} A^{V}=m_{N}$ for some $\widehat{A}$-module $N$ of dimension d. By Corollary 3.8, the Nakajima variety $\mathfrak{M}_{0}^{\bullet}\left(W^{\mathbf{d}}\right)$ is isomorphic to $\operatorname{rep}_{\mathbf{d}}(\widehat{A})$. By Theorem 3.14, the strata $\mathfrak{M}_{0}^{\bullet r e g}\left(V, W^{\mathbf{d}}\right)$ and $\mathfrak{M}_{0}^{\bullet \text { reg }}\left(V^{\prime}, W^{\mathbf{d}}\right)$ are nonempty and isomorphic to the orbits $\mathcal{O}_{N}$ and $\mathcal{O}_{N^{\prime}}$. The theorem then follows from Theorem 4.1.

\section{Acknowledgments}

The authors are grateful to B. Keller and S. Scherotzke for kindly explaining their result to us prior to its appearance on the arXiv, and to C. Geiss for sharing with us his observation which led to Corollary 3.15.

The second author was financially supported by the FQRNT.

\section{References}

[1] T. Akasaka and M. Kashiwara, Finite-dimensional representations of quantum affine algebras, Publ. RIMS Kyoto Univ. 33 (1997), 839-867. Zbl 0915.17011 MR 1607008

[2] I. Assem, D. Simson, and A. Skowroński, Elements of the representation theory of associative algebras. Vol. 1, London Math. Soc. Student Texts 65, Cambridge Univ. Press, Cambridge, 2006. Zbl 1092.16001 MR 2197389

[3] M. Auslander, Relations for Grothendieck groups of Artin algebras, Proc. Amer. Math. Soc. 91 (1984), 336-340. Zbl 0542.16028 MR 0744624

[4] M. Auslander, I. Reiten, and S. O. Smalø, Representation theory of Artin algebras, Cambridge Stud. Adv. Math. 36, Cambridge Univ. Press, Cambridge, 1997. Zbl 0834.16001 MR 1476671

[5] V. Chari and A. Pressley, Quantum affine algebras, Comm. Math. Phys. 142 (1991), 261283. Zbl 0739.17004 MR 1137064

[6] E. Frenkel and N. Reshetikhin, The $q$-characters of representations of quantum affine algebras and deformations of $W$-algebras, in Recent developments in quantum affine algebras and related topics (Raleigh, NC, 1998), Contemp. Math. 248, Amer. Math. Soc., Providence, RI, 1999, 163-205. Zbl 0973.17015 MR 1745260

[7] V. Ginzburg and É. Vasserot, Langlands reciprocity for affine quantum groups of type $A_{n}$, Int. Math. Res. Notices 1993, no. 3, 67-85. Zbl 0785.17014 MR 1208827 
[8] D. Happel, Triangulated categories in the representation theory of finite dimensional algebras, London Math. Soc. Lecture Note Ser. 119, Cambridge Univ. Press, 1988. Zbl 0635.16017 MR 0935124

[9] D. Hernandez, Algebraic approach to $q, t$-characters, Adv. Math. 187 (2004), 1-52. Zbl 1098.17009 MR 2074171

[10] D. Hernandez and B. Leclerc, Cluster algebras and quantum affine algebras, Duke Math. J. 154 (2010), 265-341. Zbl pre05788166 MR 2682185

[11] , Quantum Grothendieck rings and derived Hall algebras, arXiv:1109.0862v2 [math.QA], 2011.

[12] D. Hughes and J. Waschbüsch, Trivial extensions of tilted algebras, Proc. London Math. Soc. 46 (1983), 347-364. Zbl 0488.16021 MR 0693045

[13] B. Keller and S. Scherotzke, Graded quiver varieties and derived categories, arXiv:1303.2318 [math.RT].

[14] L. Le Bruyn and C. Procesi, Semisimple representations of quivers, Trans. Amer. Math. Soc. 317 (1990), 585-598. Zbl 0693.16018 MR 0958897

[15] G. Lusztig, Canonical bases arising from quantized enveloping algebras, J. Amer. Math. Soc. 3 (1990), 447-498. Z Zbl 0703.17008 MR 1035415

[16] , On quiver varieties, Adv. Math. 136 (1998), 141-182. Zbl 0915.17008 MR 1623674

[17] H. Nakajima, Quiver varieties and finite-dimensional representations of quantum affine algebras, J. Amer. Math. Soc. 14 (2001), 145-238. Z Zbl 0981.17016 MR 1808477

[18] , Quiver varieties and $t$-analogs of $q$-characters of quantum affine algebras, Ann. of Math. (2) 160 (2004), 1057-1097. Zbl 1140.17015 MR 2144973

[19] Quiver varieties and cluster algebras, Kyoto J. Math. 51 (2011), 71-126. Zbl 1223.13013 MR 2784748

[20] C. M. Ringel, Hall algebras and quantum groups, Invent. Math. 101 (1990), 583-591. Zbl 0735.16009 MR 1062796

[21] J. Schröer, On the quiver with relations of a repetitive algebra, Arch. Math. (Basel) 72 (1999), 426-432. Zbl 0937.16018 MR 1687516

[22] E. Vasserot, Affine quantum groups and equivariant $K$-theory, Transform. Groups 3 (1998), 269-299. Zbl 0969.17009 MR 1640675

[23] G. Zwara, Degenerations for modules over representation-finite algebras, Proc. Amer. Math. Soc. 127 (1999), 1313-1322. Zbl 0927.16008 MR 1476404 